\title{
LETTER
}

\section{High-fidelity computational simulation to refine strategies for lung-protective ventilation in paediatric acute respiratory distress syndrome}

\author{
Sina Saffaran ${ }^{1}$, Anup Das ${ }^{1}$, Jonathan G. Hardman², Nadir Yehya ${ }^{3 *}$ (D) and Declan G. Bates ${ }^{1^{*}}$
}

๑ 2019 Springer-Verlag GmbH Germany, part of Springer Nature

Dear Editor,

Mechanical ventilation in paediatric acute respiratory distress syndrome (PARDS) is less studied than in adults, with guidelines for ventilation adapted from adult ARDS. However, PARDS has a distinct epidemiology, and adult ARDS guidelines may not be appropriate in children. As an example, clinical trials suggest that lower tidal volumes $\left(V_{\mathrm{T}}\right)$ reduce mortality in adult ARDS [1]. Recent research has highlighted the potential of lung-protective strategies based on limiting driving pressure $(\Delta P)$ and mechanical power to reduce ventilator induced lung injury (VILI) [2, 3]. No trials have tested protective ventilation in PARDS, and observational studies are unclear [4]. Concerns about hypercapnia or increased dead space in paediatrics contribute to the hesitancy to lower $V_{\mathrm{T}}$. There is an urgent need for studies that can provide additional evidence regarding how lung-protective ventilation could be implemented in PARDS. We hypothesized that analysis of a large PARDS data set using a computational simulator would allow us to (a) determine the scope (in terms of lowering $V_{\mathrm{T}}, \Delta P$, and mechanical power) for safely implementing more protective ventilation; and (b) develop, test, and directly compare strategies for achieving this.

Using a prospective cohort of PARDS from the Children's Hospital of Philadelphia with detailed data

\footnotetext{
*Correspondence: yehyan@email.chop.edu; D.Bates@warwick.ac.uk ' School of Engineering, University of Warwick, Coventry CV4 7AL, UK ${ }^{3}$ Department of Anaesthesiology and Critical Care Medicine, Children's Hospital of Philadelphia, University of Pennsylvania, Philadelphia, PA, USA Full author information is available at the end of the article
}

collection (see Supplement), we developed and tested four lung-protective strategies for reducing either $V_{\mathrm{T}}$ (strategies $1-3$ ) or $\Delta P$ (strategy 4 ). Strategy 1 reduced $V_{\mathrm{T}}$ maintaining constant minute ventilation, strategy 2 reduced $V_{\mathrm{T}}$ maintaining alveolar ventilation with a fixed ratio of inspiratory time to total cycle time, strategy 3 reduced $V_{\mathrm{T}}$ maintaining alveolar ventilation with fixed inspiratory flow, and strategy 4 simultaneously reduced $V_{\mathrm{T}}$ and $\Delta P$. The simulations continued incrementally reducing $V_{\mathrm{T}}$ until safety constraints (hypoxemia, hypercarbia, peak pressure $>35 \mathrm{cmH}_{2} \mathrm{O}$, respiratory rate $[R R]>40$ breaths $/ \mathrm{min}$ ) were violated.

The simulator accurately reproduced patient data (Figs. S2 and S3) in the development cohort. Similar $V_{\mathrm{T}}$ reductions were achieved using strategies $1-3$ $(15 \%, 12 \%$, and $14 \%$; Figs. 1, S4, S5), with the number of patients being ventilated using $V_{\mathrm{T}}>10 \mathrm{~mL} / \mathrm{kg}$ falling to zero. Strategy 1 produced no significant change in mechanical power $(+1 \% ; p=0.2$, signed-rank test) but both strategies 2 and 3 resulted in increases $(+22 \%$ and $+19 \%$; both $p<0.05$ ). Strategy 4 reduced $\Delta P$ by $6 \%$ for all 30 patients in the cohort, and by $17 \%$ for the 13 patients on which this strategy could be applied without violating constraints. Strategy 4 was the only approach that produced a significant reduction in mechanical power $(-8 \%$; $p<0.05$ ). Similar trends were seen in test cohort 1 (ages $1-2$ years) and 2 (initial $V_{\mathrm{T}}>10 \mathrm{~mL} / \mathrm{kg}$ ), with test cohort 2 showing the greatest potential for lung-protective ventilation (Figs. 1, S7, S8).

Our data suggests that PARDS patients are routinely over-ventilated and there is scope for achieving protective ventilation without compromising gas exchange. 

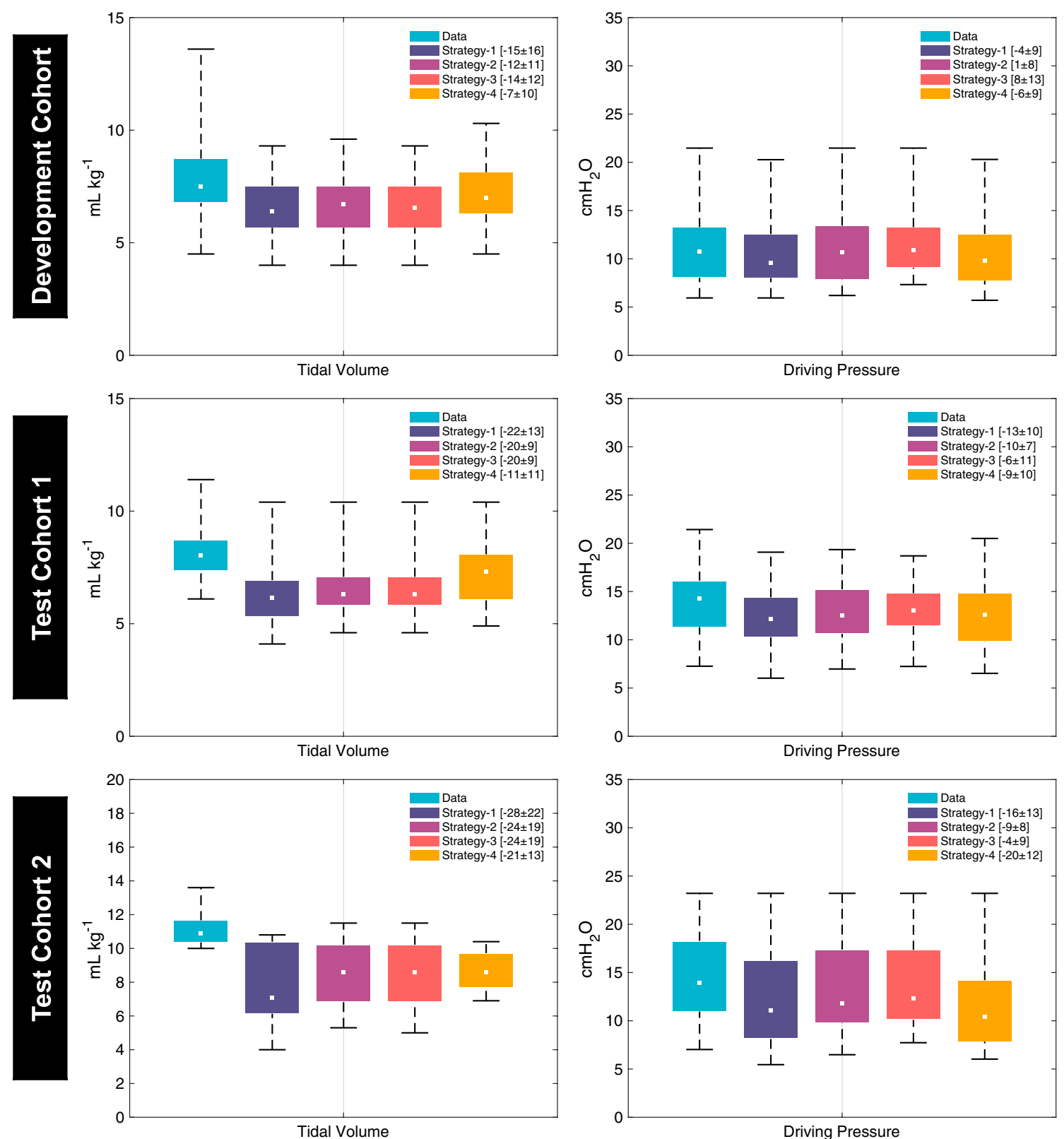

Fig. 1 Box plots show data as median (white dot), interquartile range (boxes), and full range (whiskers) of all patient data before and after implementation of different strategies. Numbers in brackets refer to percentage average change from baseline (mean \pm SD). Panels compare the amount of tidal volume (left panels) and driving pressure (right panels) changes in the development cohort, and in the two test cohorts. Test cohort 2 (initial tidal volume $>10 \mathrm{~mL} / \mathrm{kg}$ ) showed the greatest scope for potential reduction in tidal volumes and driving pressure

Such interventions could be readily implemented at the bedside by clinicians directly, or automatically via closedloop control algorithms. Our results support the design of randomized trials to better delineate the role of lungprotective ventilation in PARDS.

\section{Electronic supplementary material}

The online version of this article (https://doi.org/10.1007/s00134-019-05559-4) contains supplementary material, which is available to authorized users.

\section{Author details}

${ }^{1}$ School of Engineering, University of Warwick, Coventry CV4 7AL, UK. ${ }^{2}$ Anaesthesia and Critical Care, Division of Clinical Neuroscience, School of Medicine, University of Nottingham, Nottingham NG7 2UH, UK. ${ }^{3}$ Department of Anaesthesiology and Critical Care Medicine, Children's Hospital of Philadelphia, University of Pennsylvania, Philadelphia, PA, USA.

\section{Funding}

DGB and JGH acknowledge funding from the UK Engineering and Physical Sciences Research Council (Grant No. EP/P023444/1). NY acknowledges funding from the NIH (Grant No. NIH K23 HL-136688). 


\section{Compliance with ethical standards}

\section{Conflicts of interest}

The authors declare that they have no conflicts of interests.

\section{Ethical approval}

The study was reviewed by the CHOP Institutional Review Board, and requirement for informed consent was waived.

\section{Publisher's Note}

Springer Nature remains neutral with regard to jurisdictional claims in published maps and institutional affiliations.

Accepted: 2 February 2019

Published online: 14 February 2019

\section{References}

1. Acute Respiratory Distress Syndrome Network, Brower RG, Matthay MA, Morris A, Schoenfeld D, Thompson BT, Wheeler A (2000) Ventilation with lower tidal volumes as compared with traditional tidal volumes for acute lung injury and the acute respiratory distress syndrome. N Engl I Med 342:1301-1308

2. Amato MB, Meade MO, Slutsky AS, Brochard L, Costa EL, Schoenfeld DA, Stewart TE, Briel M, Talmor D, Mercat A, Richard JC, Carvalho CR, Brower RG (2015) Driving pressure and survival in the acute respiratory distress syndrome. N Engl J Med 372:747-755

3. Gattinoni L, Tonetti T, Cressoni M, Cadringher P, Herrmann P, Moerer O, Protti A, Gotti M, Chiurazzi C, Carlesso E, Chiumello D, Quintel M (2016) Ventilator-related causes of lung injury: the mechanical power. Intensive Care Med 42:1567-1575

4. de Jager P, Burgerhof JG, van Heerde M, Albers MJ, Markhorst DG, Kneyber MC (2014) Tidal volume and mortality in mechanically ventilated children: a systematic review and meta-analysis of observational studies. Crit Care Med 42:2461-2472 